\title{
Determination of the Fundamental Frequency of Perforated Rectangular Plates: Concentrated Negative Mass Approach for the Perforation
}

\author{
Kiran D. Mali and Pravin M. Singru \\ Department of Mechanical Engineering, Birla Institute of Technology and Science, Pilani, K.K. Birla Goa Campus, \\ Zuarinagar, Goa 403726, India
}

Correspondence should be addressed to Kiran D. Mali; malikirand@gmail.com

Received 28 February 2013; Revised 1 May 2013; Accepted 12 May 2013

Academic Editor: Joseph CS Lai

Copyright (C) 2013 K. D. Mali and P. M. Singru. This is an open access article distributed under the Creative Commons Attribution License, which permits unrestricted use, distribution, and reproduction in any medium, provided the original work is properly cited.

\begin{abstract}
This paper is concerned with a vibration analysis of perforated rectangular plates with rectangular perforation pattern of circular holes. The study is particularly useful in the understanding of the vibration of sound absorbing screens, head plates, end covers, or supports for tube bundles typically including tube sheets and support plates used in the mechanical devices. An energy method is developed to obtain analytical frequencies of the perforated plates with clamped edge, support conditions. Perforated plate is considered as plate with uniformly distributed mass. Holes are considered as concentrated negative masses. The analytical procedure using the Galerkin method is adopted. The deflected surface of the plate is approximated by the cosine series which satisfies the boundary conditions. Finite element method (FEM) results have been used to illustrate the validity of the analytical model. The comparisons show that the analytical model predicts natural frequencies reasonably well for holes of small size.
\end{abstract}

\section{Introduction}

Perforated plates are widely used in nuclear power equipments, heat exchangers, and pressure vessels. The holes in the plate are arranged in various regular penetration patterns. Industrial applications include both square and triangular array perforation patterns. Cutouts are found in mechanical, civil, marine, and aerospace structures commonly to access ports for mechanical and electrical systems, or simply to reduce weight. Cutouts are also made to provide ventilation and modify the resonant frequency of the structures. Perforated plates are often utilized as head plates, end covers, or supports for tube bundles typically including tube sheets and support plates.

Many studies have been done on perforated plates having rectangular/square and triangular array of holes, especially [1-3] dealing with equivalent properties of material for perforated plate. These equivalent material properties are used in vibration analysis to consider perforated plate as full solid plate. Burgemeister and Hansen [4] showed that, to predict accurately the resonance frequencies of simply supported perforated panel, effective material constants cannot be used in classical equations. They used cubic function fitted from ANSYS results to determine the effective resonance frequency ratio for large range of panel geometries with an error of less than 3\%. Mali and Singru [5] introduced concept of concentrated negative masses for perforation holes and determined fundamental frequency of rectangular plate carrying four circular perforations in rectangular pattern. Mali and Singru formulated an analytical model by using unit step functions [6] and the greatest integer functions [7] to express nonhomogeneity in Young's modulus and density and determined fundamental frequency of free vibration of perforated plate.

Low $[8,9]$ determined the vibration frequencies of rectangular plates with weights mounted at various locations. $\mathrm{He}$ also developed improved model to determine the frequency of vibrating plates carrying multiple masses at various positions. Low et al. $[10,11]$ obtained natural frequencies of rectangular plates carrying a single and multiple concentrated masses by using Rayleigh-Ritz method for different boundary conditions. They compared results obtained from 
the analytical study using the energy method with those measured experimentally. Boay [12] analyzed free vibration analysis of rectangular isotropic plates carrying a concentrated mass. The Ritz approach is applied to rectangular plates with various edge support combinations of clamp and simple support conditions. The effect of different locations of the concentrated mass on the fundamental frequency of the plate is presented in detail. Wu and Luo [13] determined the natural frequencies and the corresponding mode shapes of a uniform rectangular flat plate carrying any number of point masses and translational springs by means of the analytical and numerical combined method (ANCM). Avalos et al. [14] studied transverse vibrations of simply supported rectangular plates with rectangular cutouts carrying an elastically mounted concentrated mass. Ostachowicz et al. [15] presented new results for the identification of the location of a concentrated mass on isotropic plates by means of a genetic algorithm search technique based on changes in natural frequencies. $\mathrm{Li}$ [16] presented an exact approach for free vibration of an isotropic rectangular plate carrying a line-concentrated mass and with a line-translational spring support or carrying a line-spring-mass system. Altintas [17] investigated the special behaviors of linear vibrating plates with special parameters near degenerate modes. The special parameters considered in the study are the location and quantity of an additional mass, which have an effect on removing the system symmetry. Zhang [18] presented and compared different methods on the eigenfrequency computation of a beam and a plate carrying arbitrary number of concentrated mass/spring. The advantages and disadvantages of these methods are analyzed and discussed. Amabili [19] studied nonlinear forced vibrations of rectangular plates carrying a central concentrated mass. Amabili et al. [20, 21] studied effect of concentrated masses with rotary inertia on vibrations of rectangular plates and large-amplitude vibrations of rectangular plates carrying concentrated masses.

From the literature on vibration of perforated plates, authors found that, there is no evidence on the analytical formulation by considering negative mass approach for holes except for reference [5] where authors have studied only four specimens of rectangular plates with four circular perforations. They have not discussed the limiting condition of the approach for obtaining the accuracy in predicted value of fundamental frequency. In this paper, free vibration of perforated plate with rectangular perforation pattern of nine circular perforations is studied. Boundary condition used for the plate is clamped on all four edges. In the current approach Galerkin method is used for determining the fundamental frequency of rectangular perforated plate with rectangular perforation pattern of circular holes. Perforated plate is considered as plate with uniformly distributed mass, and holes are considered as concentrated negative masses. The deflected surface of the plate is approximated by the cosine series which satisfies the boundary conditions. Ten specimens are analyzed numerically with different perforation diameters to obtain fundamental frequency. To validate the proposed model FEM results have been used. Percentage error in fundamental frequency obtained by comparing numerical and FEM results is plotted against ratio of plate area $(A)$ to perforation hole area $(A p)$. For the variation of the percent error in the fundamental frequency, trendline is established by regression analysis. Further from the equation of the trendline limiting condition in terms of the ratio of plate area $(A)$ to perforation hole area $(A p)$, to obtain accuracy in fundamental frequency is obtained. Present analytical model is thus more useful for predicting accurately the fundamental frequencies of wide range of small size perforation geometries, for rectangular plates with rectangular penetration pattern for all edges clamped support condition.

\section{Analytical Formulations}

2.1. Equation of Motion for Isotropic Rectangular Plates. The Galerkin method is applied to the plate vibration problems $[5,22]$ to get the equation of motion. The partial differential equation governing the free transverse vibration of an isotropic thin plate with dimensions $a, b$ shown in Figure 1 is [22]

$$
\begin{gathered}
D\left(\frac{\partial^{4} w}{\partial x^{4}}+2 \frac{\partial^{2} w}{\partial x^{2}} \frac{\partial^{2} w}{\partial y^{2}}+\frac{\partial^{4} w}{\partial y^{4}}\right)+\rho h \frac{\partial^{4} w}{\partial t^{2}}=0, \\
D \nabla^{2} \nabla^{2} w(x, y, t)+\rho h \frac{\partial^{4} w}{\partial t^{2}}(x, y, t)=0,
\end{gathered}
$$

where $h$ is the uniform plate thickness, $\rho$ is the density, $w$ is transverse displacement, $D$ is the flexural rigidity, and $\nabla^{2}$ is two-dimensional Laplacian operator. $D$ and $\nabla^{2}$ are given as $[22,23]$

$$
D=\frac{E h^{3}}{12\left(1-v^{2}\right)}, \quad \nabla^{2}=\frac{\partial^{2}}{\partial x^{2}}+\frac{\partial^{2}}{\partial y^{2}},
$$

where $E$ is modulus of elasticity and $v$ is Poisson's ratio.

To solve (2) and obtain $w(x, y, t)$ in general, the following solution can be assumed [22]:

$$
w(x, y, t)=(A \cos \omega t+B \cos \omega t) W(x, y) .
$$

This is separable solution of the shape function $W(x, y)$ describing the modes of the vibration and some harmonic function of time; $\omega$ is the natural frequency of the plate vibration which is related to vibration period $T$ by the relationship

$$
\omega=\frac{2 \pi}{T} .
$$

Introducing (4) into (2) we get [22]

$$
D \nabla^{2} \nabla^{2} w(x, y)-\omega^{2} \rho h W=0 .
$$

The shape function satisfying the boundary conditions for rectangular plate with dimensions $a$ and $b$ is assumed in the form of series as [22]

$$
W(x, y)=\sum_{i=1}^{n} C_{i} W_{i}(x, y),
$$

where $C_{i}$ are unknown coefficients.

Now following the general procedure of the Galerkin method [22] the unknown coefficients $C_{i}$ can be determined from orthogonality conditions. 


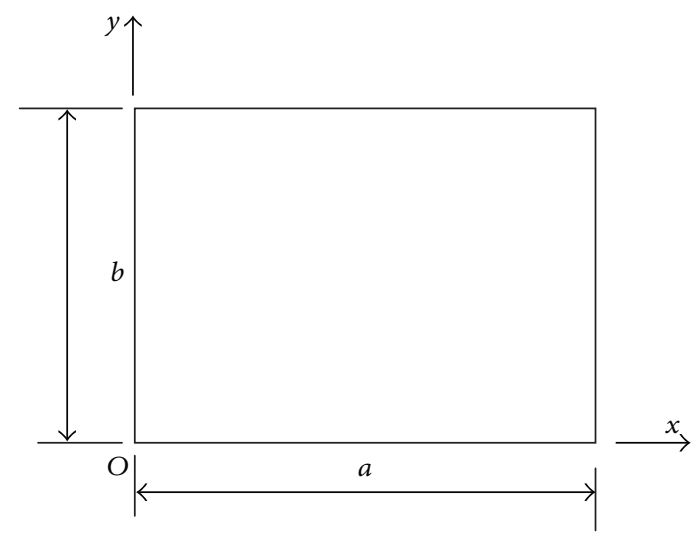

FIGURE 1: Rectangular plate coordinates.

For the plate problem vibration given by (6), the orthogonality conditions together with (7) result in (8) as given below [22].

$$
\begin{array}{r}
\iint_{A}\left(D \sum_{i=1}^{n} C_{i} \nabla^{2} \nabla^{2} W_{i}-\omega^{2} \rho h \sum_{i=1}^{n} C_{i} W_{i}\right) \\
W_{k} d x d y=0 \\
k=1,2, \ldots, n
\end{array}
$$

The above conditions when implemented numerically leads to the Galerkin system of linear algebraic equations of the form

$$
\begin{gathered}
a_{11} C_{1}+a_{12} C_{2}+\cdots=0 \\
\vdots \\
a_{n 1} C_{1}+a_{n 2} C_{2}+\cdots=0,
\end{gathered}
$$

where

$$
a_{i k}=a_{k i}=\iint_{A}\left[D \nabla^{2} \nabla^{2} W_{i}-\omega^{2} \rho h W_{i}\right] W_{k} d x d y .
$$

This system of homogeneous equations has a nontrivial solution if its determinant $\Delta(\omega)$ made up of the coefficients $a_{i k}$ is equal to zero. Therefore we obtain $n$th order characteristic equation for determining the natural frequencies [22].

$$
\Delta(\omega)=0 .
$$

This equation will have an infinite number of solutions which constitute the frequency spectrum for the given plate. In general, the frequencies will depend on two parameters: $m$ and $n(m=1,2, \ldots ; n=1,2, \ldots)$. The lowest frequency is called the frequency of the fundamental mode or the fundamental natural frequency, and all other frequencies are called the frequencies of higher harmonics. For each frequency $\omega_{m n}$, there is a corresponding shape function $W(x, y)$, which on the basis of the homogeneous equations, is determined by a constant multiplier (which can be assumed as being equal to unity).
2.2. Determination of the Fundamental Frequency of Plates with Perforations. A rectangular plate with coordinate system $(O ; x, y, z)$, having the origin $O$ at one corner, is considered as shown in Figure 2. Coordinates of the plate clamped on all edges carrying circular holes. The displacement of an arbitrary point of coordinates $(x, y)$ on the middle surface of the plate is denoted by $W$, in out-of-plane $(z)$ direction. The boundary conditions considered here are all edges clamped. Geometric parameters of the rectangular plate are $a$ and $b$ for sides, $h$ for thickness and $d$ is hole diameter which for uniform for all perforations. The assumptions made in the following formulation are that transverse defections are small so that the dynamic behavior of the plate is governed by classical thin plate theory. Mass of the plate $(m)$ is considered as uniformly distributed with nine concentrated negative masses denoted by " $-M$." The negative sign indicates that the concentrated mass " $-M$ " cancels out the effect of the same amount of mass of the homogenous plate at the position of the cutouts. This is an equivalent approach to apply the Galerkin method for the given perforated plate vibration problem. Analytical model in the present work does not consider any rotary inertia of the plate.

The middle surface displacement is approximated by using shape function $W(x, y)$ in the form of a series, which satisfies the boundary conditions on the edges $x=0, x=a$ and $y=0, y=b$. Let us represent the shape function $W(x, y)$ for a rectangular plate with dimensions $a$ and $b$ in the form [22]

$$
W(x, y)=\sum_{i} \sum_{k} C_{i k} W_{i k}(x, y)
$$

where $C_{i k}$ are unknown coefficients representing the amplitudes of the free vibration modes and $W_{i k}(x, y)$ is the product of the pertinent eigenfunctions of lateral beam vibrations. Consider

$$
W_{i k}(x, y)=F_{i}(x) F_{k}(y)
$$

which satisfy the prescribed boundary conditions on the edges $x=0, x=a$ and $y=0, y=b$. In (13) $F_{i}(x)$ and $F_{k}(y)$ represent the $i$ th and $k$ th modes of freely vibrating beams of spans $a$ and $b$, respectively.

By applying Galerkin method for determining fundamental frequency, (10) can be modified for the coefficients $a_{i k}$ of Galerkin's system of equations, to the present problem, as follows [22]:

$$
\begin{aligned}
a_{i k}=a_{k i}= & \iint_{A}\left[D \nabla^{2} \nabla^{2} W_{i}-\omega^{2} \rho h W_{i}\right) W_{k} d x d y \\
& -\sum M \omega^{2} W_{i} W_{k} .
\end{aligned}
$$

The deflected surface of the vibrating plate is approximated by the series

$$
W(x, y)=\sum_{i=1}^{\infty} \sum_{k=1}^{\infty} C_{i k}\left(1-\cos \frac{i 2 \pi x}{a}\right)\left(1-\cos \frac{k 2 \pi y}{b}\right) .
$$

This satisfies the boundary condition clamped on all edges. 


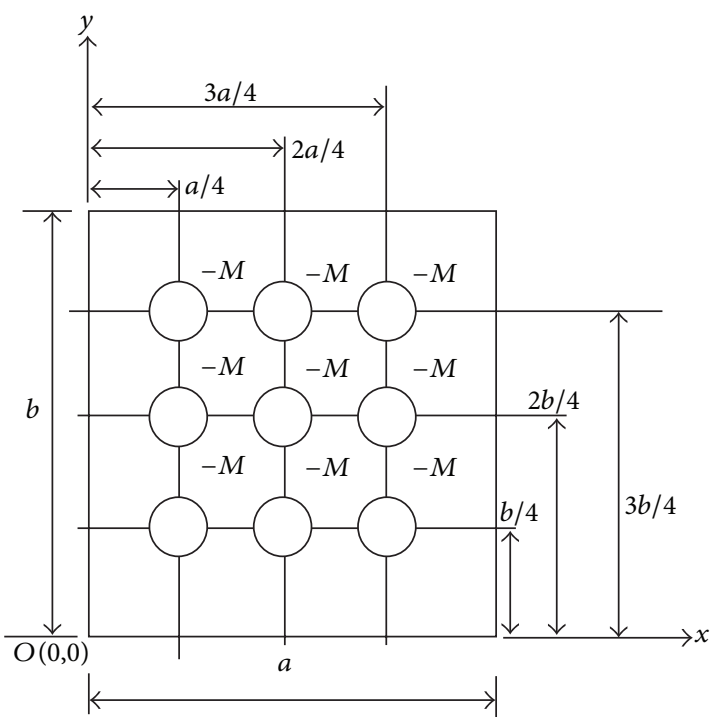

Figure 2: Coordinates of the plate clamped on all edges carrying circular holes.

For the first approximation retaining only first term in the in the expansion of (15) we obtain from (14)

$$
\begin{array}{r}
a_{11}=\int_{0}^{a} \int_{0}^{b}\left[D\left(\frac{\partial^{4} W_{1}}{\partial x^{4}}+2 \frac{\partial^{2} W_{1}}{\partial x^{2}} \frac{\partial^{2} W_{1}}{\partial y^{2}}+\frac{\partial^{4} W_{1}}{\partial y^{4}}\right)\right. \\
\left.-m \omega^{2} W_{1}\right] W_{1} d x d y-\sum M \omega^{2} W_{1}^{2} .
\end{array}
$$

Introducing in (16) $W_{1}$,

$$
W_{1}=\left(1-\cos \frac{2 \pi x}{a}\right)\left(1-\cos \frac{2 \pi y}{b}\right) .
$$

After simplification of (16) the following expressions are obtained:

$$
\begin{gathered}
D\left(\frac{12 b \pi^{4}}{a^{3}}+\frac{12 a \pi^{4}}{b^{3}}+\frac{8 a \pi^{4}}{a b}\right)-m \omega^{2}\left(\frac{9 a b}{4}\right)+32 M \omega^{2}=0, \\
\omega=\frac{\left.-4 \pi^{2} \sqrt{-a b(-9 m a b+128 M) D\left(3 b^{4}\right.}+3 a^{4}+2 a^{2} b\right)}{a^{2} b^{2}(-9 m a b+128 M)} .
\end{gathered}
$$

\section{Numerical Simulation}

Analytical model developed in Section 2.2 is applicable to rectangular perforated plates with different side dimensions, provided that the perforations are of the same size. By virtue of the symbolic forms presented in this work, the method can be applied to analytical studies of perforated plates with different boundary conditions. To estimate the sensitivity of the method for various cases of hole sizes and plate sizes, numerical analysis is carried out for ten different plate
TABLE 1: Specimen parameters.

\begin{tabular}{lccccc}
\hline Specimen no. & $a(\mathrm{~mm})$ & $b(\mathrm{~mm})$ & $h(\mathrm{~mm})$ & $d(\mathrm{~mm})$ & $\left(A / A_{p}\right)$ \\
\hline 1 & 138 & 216 & 2 & 5 & 1518.154 \\
2 & 138 & 216 & 2 & 10 & 379.5384 \\
3 & 138 & 216 & 2 & 15 & 168.6837 \\
4 & 138 & 216 & 2 & 25 & 60.72615 \\
5 & 138 & 216 & 2 & 30 & 42.17094 \\
6 & 276 & 432 & 2 & 10 & 1518.154 \\
7 & 276 & 432 & 2 & 20 & 379.5384 \\
8 & 276 & 432 & 2 & 25 & 242.9046 \\
9 & 276 & 432 & 2 & 50 & 60.72615 \\
10 & 276 & 432 & 2 & 65 & 35.93263 \\
\hline
\end{tabular}

specimens listed in Table 1. Last column of Table 1 lists the ratio of the plate area $(A)$ to the area of perforation hole $\left(A_{p}\right)$

$$
\begin{aligned}
& A=a \times b, \\
& A_{p}=\frac{\pi d^{2}}{4} .
\end{aligned}
$$

All the specimens considered have thickness $2 \mathrm{~mm}$. Coordinates of the perforation centers $(x \mathrm{~mm}, y \mathrm{~mm})$ are given in Table 2 . The following are the material properties [24] considered for all specimen plates analyzed:

$$
\begin{gathered}
E=2.1 \times 10^{11} \mathrm{~N} / \mathrm{m}^{2}, \\
v=0.3, \\
\rho=7850 \mathrm{~kg} / \mathrm{m}^{3} .
\end{gathered}
$$

\section{Finite Element Method (FEM) Analysis}

The proposed analytical model is validated by comparing the numerical analysis results with FEM modal analysis results. FEM modal analysis is carried out by ANSYS 11 using Shell 63 element. Parameters of the plate specimen considered in this study are shown in Table 1. Analysis is carried out for clamped steel plates having $2 \mathrm{~mm}$ thickness and carrying nine holes at positions shown in Figure 2. Meshing is done by free meshing with smart size option, and quadrilateral elements are used. Mesh convergence for FEM results is checked for every specimen. This is checked by running different simulations. Final solution is chosen based on the mesh quality as well as mesh size. Thus converged solution is given in Table 3. It is assumed that structure is formed of isotropic homogeneous elastic material, that is, structural steel with material properties [24] same as that used in numerical analysis.

\section{Results and Discussions}

Comparison of numerical and FEM natural frequencies of the plate specimens for first mode is given in Table 3. Last column of Table 3 shows percentage error in fundamental frequency values obtained by numerical analysis and by FEM analysis. The agreement between the analytical approach and the finite 
TABLE 2: Coordinates of the perforation centers.

\begin{tabular}{|c|c|c|c|c|c|c|c|c|c|}
\hline Specimen size $(\mathrm{mm} \times \mathrm{mm})$ & & & Coor & inates of th & perforation & centers $(x \mathrm{~m}$ & , $y \mathrm{~mm})$ & & \\
\hline$a \times b$ & $a / 4, b / 4$ & $a / 4,2 b / 4$ & $a / 4,3 b / 4$ & $2 a / 4, b / 4$ & $2 a / 4,2 b / 4$ & $2 a / 4,3 b / 4$ & $3 a / 4, b / 4$ & $3 a / 4,2 b / 4$ & $3 a / 4,3 b / 4$ \\
\hline $138 \times 216$ & $34.5,54$ & $34.5,108$ & $34.5,162$ & 69,54 & 69,108 & 69,162 & $103.5,54$ & $103.5,108$ & $103.5,162$ \\
\hline $276 \times 432$ & 69,108 & 69,216 & 69,324 & 138,108 & 138,216 & 138,324 & 207,108 & 207,216 & 207,324 \\
\hline
\end{tabular}

TABLE 3: Fundamental frequency results of numerical and FEM simulations.

\begin{tabular}{lccccc}
\hline $\begin{array}{l}\text { Specimen } \\
\text { no. }\end{array}$ & $\begin{array}{c}d \\
(\mathrm{~mm})\end{array}$ & $\left(\mathrm{A} / \mathrm{A}_{p}\right)$ & $\begin{array}{c}\omega, \text { Numerical } \\
(\mathrm{Hz})\end{array}$ & $\begin{array}{c}\omega, \mathrm{FEM} \\
(\mathrm{Hz})\end{array}$ & $\%$ Error \\
\hline \multicolumn{5}{c}{ Specimens with dimensions $(138 \mathrm{~mm} \times 216 \mathrm{~mm})$} \\
\hline 2 & 5 & 1518.154 & 718.63 & 693.38 & 3.64 \\
3 & 10 & 379.5384 & 729.04 & 693.85 & 5.07 \\
4 & 15 & 168.6837 & 747.46 & 695.97 & 7.40 \\
5 & 25 & 60.72615 & 817.34 & 710.17 & 15.09 \\
\hline \multicolumn{6}{c}{ Specimens with dimensions $(276 \mathrm{~mm} \times 432 \mathrm{~mm})$} \\
\hline 6 & 10 & 1518.154 & 179.65 & 173.37 & 3.62 \\
7 & 20 & 379.5384 & 182.26 & 173.46 & 5.07 \\
8 & 25 & 242.9046 & 184.29 & 173.65 & 6.13 \\
9 & 50 & 60.72615 & 204.33 & 177.53 & 15.10 \\
10 & 65 & 35.93263 & 230.04 & 183.55 & 25.33 \\
\hline
\end{tabular}

element results is excellent when perforation size is small. The maximum difference is of the order $25.33 \%$ especially for the specimen having $A / A_{p}=35.93263$. Figure 3 shows the variation of the percent error in fundamental frequency with respect to $A / A_{p}$ ratio. Dotted line shows the trendline for this variation. The power trendline clearly demonstrates the decrease in percentage error with increase in $A / A_{p}$ ratio. Equation used to calculate the least squares fit through points for the trendline is

$$
\% \text { Error }=f\left(\frac{A}{A_{p}}\right)^{g} .
$$

In the above expression $f$ and $g$ are constants. For the percentage error, $A / A_{p}$ ratio data given in Table 3 values of constants $f$ and $g$ are found to be 123.97 and -0.51 , respectively. Thus equation of the trendline shown in Figure 3 becomes

$$
y=123.97 x^{-0.51},
$$

where $y$ and $x$ are values of percentage error and $A / A_{p}$ ratio, respectively.

$R$-squared value shown in Figure 3 is a correlation coefficient which reveals how closely the estimated values for the trendline correspond to actual data

$$
R^{2}=0.9371
$$

It is observed that percent error increases above $10 \%$, when $A / A_{p}$ ratio decreases below the value of 139.2380 . Thus present approach has limitation in predicting the value of

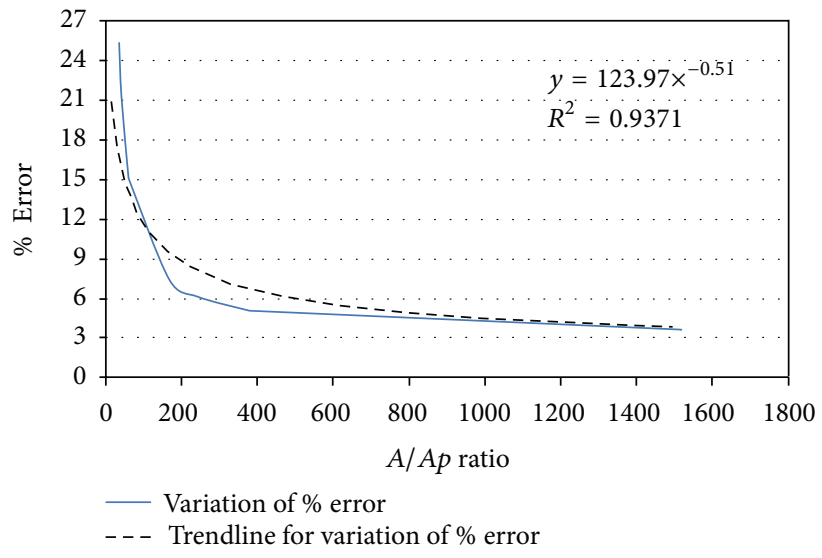

FIGURE 3: Variation of the percent error in fundamental frequency with $A / A_{p}$ ratio.

fundamental frequency, as it is based on the basic assumption of concentrated negative mass. Further from Table 3 it is observed that for specimens of different configurations but having the same $A / A_{p}$ ratio analytical model gives results with the same accuracy (specimens 1, 6 and 2,7).

When perforation size increases, results obtained by proposed method deviate more. This occurs because, as perforation size increases, it changes stiffness also significantly, but proposed model considers only change in/reduction in the mass or kinetic energy but not strain energy. Due to this unaccounted change in strain energy, fundamental frequencies obtained by this method are higher than FEM results. Thus deviation between numerical and FEM fundamental frequencies becomes more as perforation size increases.

The reasonably good performance of the proposed model, for small holes is demonstrated in Table 3 and Figure 3. Variation of the percent error in fundamental frequency with $A / A_{p}$ ratio is due to the fact that the effects of both the different holes and their locations on the frequency have been accounted.

\section{Conclusions}

This work presents an analytical model to estimate the fundamental frequency of uniform thickness plates carrying circular perforations in rectangular pattern. The effect of perforation on the natural frequency of plate has been modeled using concept of concentrated negative mass in the Galerkin method. The proposed model has been verified by comparing the numerical results with FEM results. It is found that the error in the fundamental frequency is of the order of $5 \%$ when ratio of the area of plate $(A)$ to the area of single perforation 
$\left(A_{p}\right)$ is 542.0170. It is found that the error in the fundamental frequency is more when perforation size increases. Thus, fundamental frequency of perforated plate can be obtained for small size of the perforation by a proper choice of the plate parameters and shape function depending on the boundary condition.

\section{References}

[1] W. J. O'Donnell, "Effective elastic constants for the bending of thin perforated plates with triangular and square penetration patterns," Journal of Engineering for Industry, vol. 95, no. 1, pp. 121-128, 1973.

[2] S. Choi, K. H. Jeong, T. Kim, K. Kim, and K. Park, "Free vibration analysis of perforated plates using equivalent elastic properties," Journal of the Korean Nuclear Society, vol. 30, no. 5, pp. 416-423, 1998.

[3] W. C. Wang and K. H. Lai, "Hybrid determination of equivalent characteristics of perforated plates," Experimental Mechanics, vol. 43, no. 2, pp. 163-172, 2003.

[4] K. A. Burgemeister and C. H. Hansen, "Calculating resonance frequencies of perforated panels," Journal of Sound and Vibration, vol. 196, no. 4, pp. 387-399, 1996.

[5] K. D. Mali and P. M. Singru, "Determination of the fundamental frequency of perforated plate with rectangular perforation pattern of circular holes by negative mass approach for the perforation," International Journal of Advanced Materials Manufacturing and Characterization, vol. 1, no. 1, pp. 105-109, 2012.

[6] K. D. Mali and P. M. Singru, "An aanalytical model to determine fundamental frequency of free vibration of perforated plate by using unit step functions to express non homogeneity," Journal of Vibroengineering, vol. 14, no. 3, pp. 1292-1298, 2012.

[7] K. D. Mali and P. M. Singru, "An analytical model to determine fundamental frequency of free vibration of perforated plate by using greatest integer functions to express non homogeneity," Advanced Materials Research, vol. 622, pp. 600-604, 2013.

[8] K. H. Low, "Analytical and experimental investigation on a vibrating rectangular plate with mounted weights," Journal of Sound and Vibration, vol. 160, no. 1, pp. 111-121, 1993.

[9] K. H. Low, "Improved model for the frequency estimate of massloaded plates by a combined use of equivalent center mass and stiffness factors," International Journal of Mechanical Sciences, vol. 43, no. 2, pp. 581-594, 2001.

[10] K. H. Low, G. B. Chai, and G. S. Tan, "A comparative study of vibrating loaded plates between the Rayleigh-Ritz and experimental methods," Journal of Sound and Vibration, vol. 199, no. 2, pp. 285-297, 1997.

[11] K. H. Low, G. B. Chai, T. M. Lim, and S. C. Sue, "Comparisons of experimental and theoretical frequencies for rectangular plates with various boundary conditions and added masses," International Journal of Mechanical Sciences, vol. 40, no. 11, pp. 11191131, 1998.

[12] C. G. Boay, "Frequency analysis of rectangular isotropic plates carrying a concentrated mass," Computers and Structures, vol. 56, no. 1, pp. 39-48, 1995.

[13] J. S. Wu and S. S. Luo, "Use of the analytical-and-numericalcombined method in the free vibration analysis of a rectangular plate with any number of point masses and translational springs," Journal of Sound and Vibration, vol. 200, no. 2, pp. 179194, 1997.

[14] D. R. Avalos, H. A. Larrondo, P. A. A. Laura, and R. E. Rossi, "Transverse vibrations of simply supported rectangular plates with rectangular cutouts carrying an elastically mounted concentrated mass," Journal of Sound and Vibration, vol. 202, no. 4, pp. 585-592, 1997.

[15] W. Ostachowicz, M. Krawczuk, and M. Cartmell, "The location of a concentrated mass on rectangular plates from measurements of natural vibrations," Computers and Structures, vol. 80, no. 16-17, pp. 1419-1428, 2002.

[16] Q. S. Li, "An exact approach for free vibration analysis of rectangular plates with line-concentrated mass and elastic linesupport," International Journal of Mechanical Sciences, vol. 45, no. 4, pp. 669-685, 2003.

[17] G. Altintas, "Effect of mass based imperfections on behavior of linear vibrating plates near degenerate modes," Journal of Vibration and Control, vol. 15, no. 2, pp. 219-231, 2009.

[18] Y. Zhang, "Eigenfrequency computation of beam/plate carrying concentrated mass/spring," Journal of Vibration and Acoustics, vol. 133, no. 2, Article ID 021006, 10 pages, 2011.

[19] M. Amabili, "Geometrically nonlinear vibrations of rectangular plates carrying a concentrated mass," Journal of Sound and Vibration, vol. 329, no. 21, pp. 4501-4514, 2010.

[20] M. Amabili, M. Pellegrini, F. Righi, and F. Vinci, "Effect of concentrated masses with rotary inertia on vibrations of rectangular plates," Journal of Sound and Vibration, vol. 295, no. 1-2, pp. 1-12, 2006.

[21] M. Amabili and S. Carra, "Experiments and simulations for large-amplitude vibrations of rectangular plates carrying concentrated masses," Journal of Sound and Vibration, vol. 331, no. 1, pp. 155-166, 2012.

[22] E. Ventsel and T. Krauthammer, Thin Plates and Shells: Theory, Analysis, and Applications, Marcel Dekker, New York, NY, USA, 2001.

[23] S. Chakraverty, "Vibration basics for plates," in Vibration of Plates, CRC Press, Taylor \& Francis Group, Boca Raton, Fla, USA, 1st edition, 2009.

[24] A. E. Armenakas, "Appendix 'A' mechanical properties of materials," in Advanced Mechanics of Materials and Applied Elasticity, CRC Press, Boca Raton, Fla, USA, 2006. 

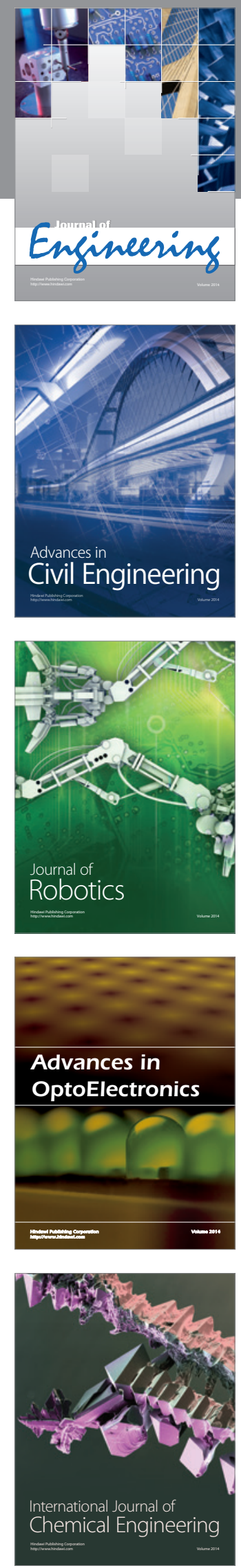

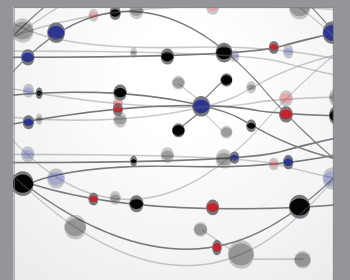

The Scientific World Journal
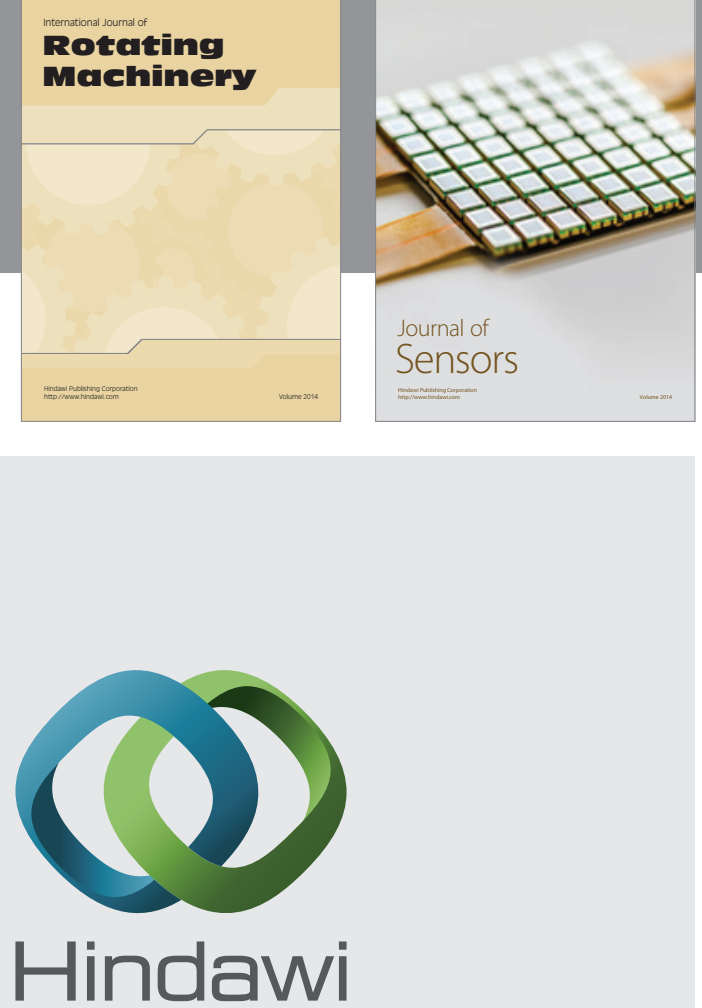

Submit your manuscripts at http://www.hindawi.com
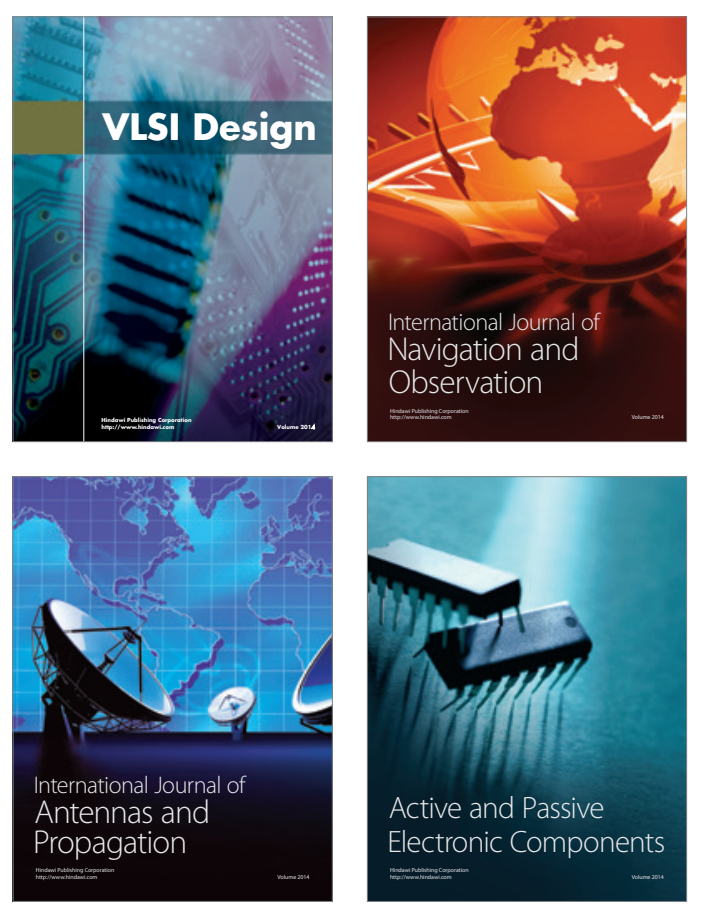
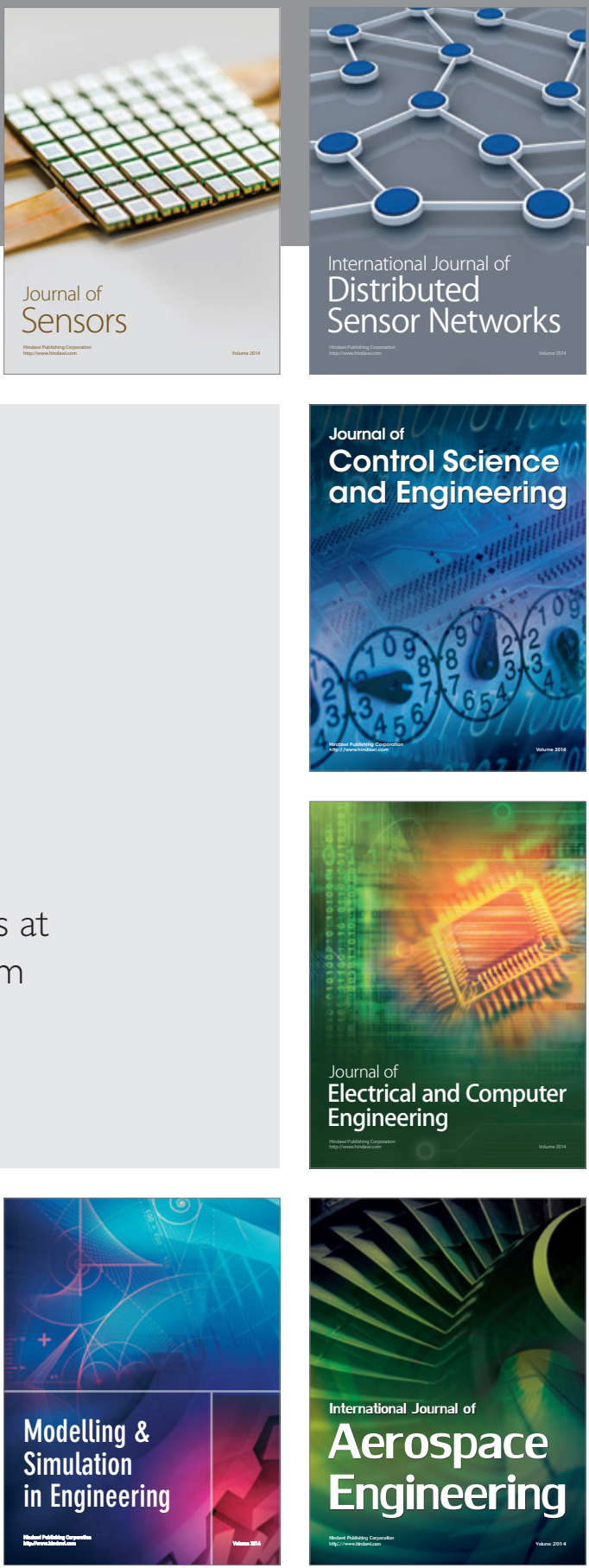

Journal of

Control Science

and Engineering
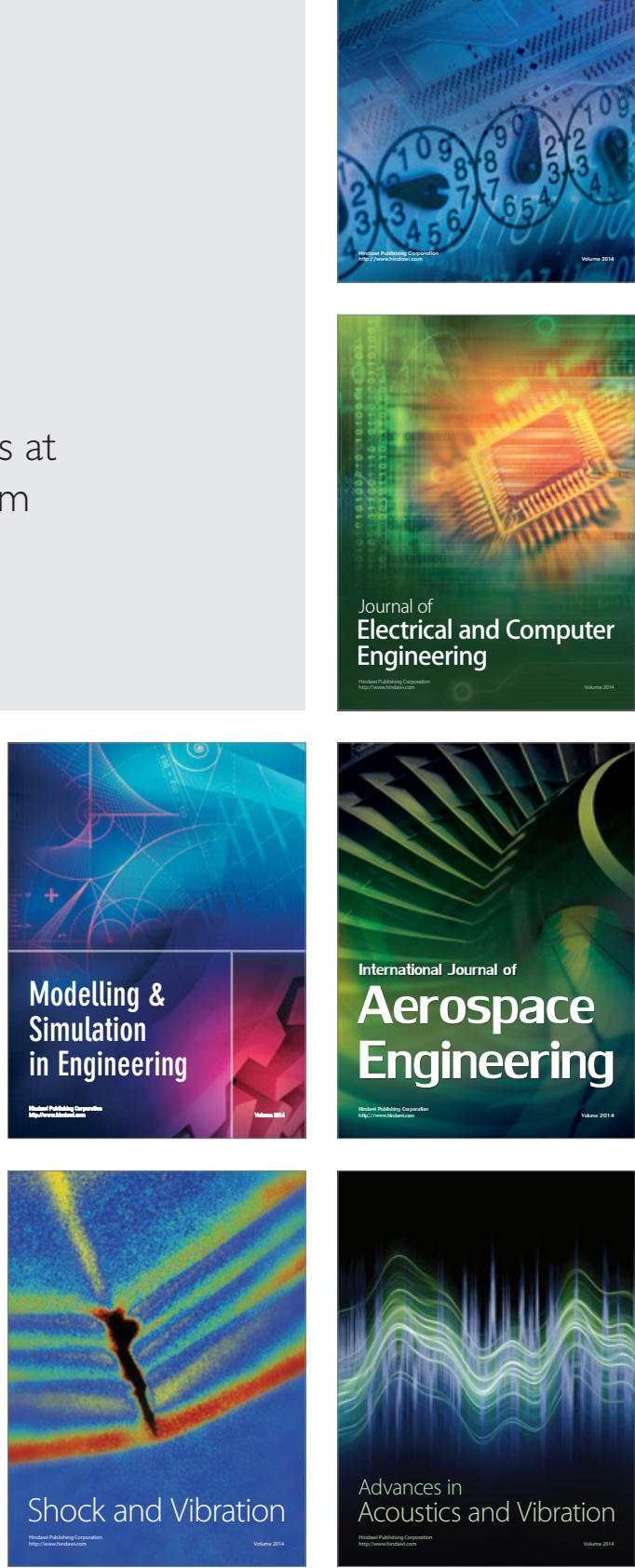